\title{
Otimização e validação de um método enzimático para a determinação de glicose em tubérculos de batata
}

\author{
Optimization and validation of an enzymatic method to quantify glucose in potato tubers
}

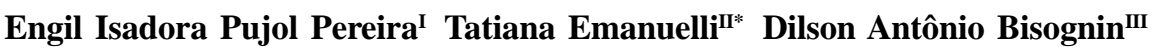 \\ Sérgio Tonetto de Freitas ${ }^{\mathrm{IV}}$
}

RESUMO

\begin{abstract}
Os tubérculos de batata destinados ao processamento devem apresentar coloração clara após a fritura, o que é condicionado pelos baixos teores de açúcares redutores, glicose e frutose. Para auxiliar os programas de melhoramento na seleção de clones com baixos teores destes açúcares, o objetivo deste trabalho foi adaptar e validar uma metodologia simples, econômica e eficiente para quantificar o teor de glicose em tubérculos de batata utilizando um kit laboratorial enzimático desenvolvido para uso em amostras de sangue e urina. Os procedimentos de validação seguiram as recomendações do INMETRO dispostas no documento DOQ-CGCRE-008 de 2003. O

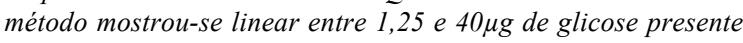
na amostra, sendo que o limite de quantificação foi $0,319 \mathrm{mg}$ $g^{-1}$ de massa fresca de batata. A faixa linear de trabalho do método foi desde o limite de quantificação até $1,32 \mathrm{mg}^{-1}$ de massa fresca de batata. A recuperação média da extração foi de 99,0\%. O método proposto foi aplicado para determinar o teor de glicose em amostras de batata "Macaca", que variou de 1,4 a 2,9mg $\mathrm{g}^{-1}$ massa fresca de batata.
\end{abstract}

Palavras-chave: Solanum tuberosum L., qualidade de tubérculo, glicose, kit enzimático, espectrofotometria.

\section{ABSTRACT}

The processing industry requires potato tubers with low levels of reduced sugars, glucose and fructose, which is responsible for the whiteness of the chip color. The objective of this research was to optimize and validate a simple, inexpensive, and precise enzymatic method to quantify glucose in potato tubers. This method will be useful in breeding programs to select clones for processing purposes with low levels of glucose.
The validation procedure followed the recommendations described in INMETRO document DOQ-CGCRE-008 in 2003. The method was linear between 1.25 to $40 \mu \mathrm{g}$ of glucose in the sample. The quantification limit was $0.319 \mathrm{mg}^{-1}$ of potato fresh weight. The linear working interval of the method started at the quantification limit and ended at $1.32 \mathrm{mg} \mathrm{g}^{-1}$ of potato fresh weight. The average recovery in the extraction was $99.0 \%$. The proposed method was used to determine glucose level in samples of 'Macaca' tubers, which ranged from 1.4 to $2.9 \mathrm{mg}$ $\mathrm{g}^{-1}$ potato fresh weight.

Key words: Solanum tuberosum L., tuber quality, glucose, enzymatic kit, spectrophotometry.

\section{INTRODUÇÃO}

Parte da produção da batata (Solanum tuberosum L.) é destinada ao processamento industrial na forma de batata chips ou pré-frita congelada. A cor clara desses produtos após a fritura é um dos parâmetros de qualidade exigidos pelo consumidor e é condicionada pelo baixo teor de açúcares redutores (glicose e frutose) presente no tubérculo (MARQUEZ \&AÑON, 1986). Embora ambos os açúcares apresentem alta correlação com a cor dos chips, a glicose tem maior associação com o escurecimento (BROWN et al, 1990).

Durante a fritura de batatas com elevados teores de açúcares redutores, o grupamento carbonila destes açúcares reage com aminoácidos livres presentes no citoplasma, formando produtos de coloração marrom

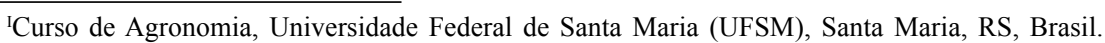

IINúcleo Integrado de Desenvolvimento em Análises Laboratoriais (NIDAL), Departamento de Tecnologia e Ciência de Alimentos, Centro de Ciências Rurais (CCR), UFSM. Faixa de Camobi, Km 9, Campus Universitário, 97105-900, Santa Maria, RS, Brasil. Email: tatiemanuelli@smail.ufsm.br. *Autor para correspondência.

IIIDepartamento de Fitotecnia, CCR, UFSM, Santa Maria, RS, Brasil.

IVPlant Biology Graduate Program, University of California, Davis, USA. 
(melanoidinas) e desenvolvendo sabor amargo, indesejável aos chips e às fritas (COELHO et al., 1999; DAVIDS et al., 2004).

Os programas de melhoramento da batata buscam selecionar genótipos que apresentem, entre outras características, baixos teores de açúcares redutores. Essa informação pode ser obtida não só por meio da determinação do conteúdo total de açúcares redutores, mas também pela quantificação da glicose ou frutose nos tubérculos (PEREIRA et al., 1994). Entretanto, a escolha de uma metodologia adequada para tais análises deve considerar aspectos como o custo de aquisição de equipamentos e reagentes, assim como a praticidade e a confiabilidade da técnica.

As determinações de açúcares redutores por métodos espectrofotométricos como o método descrito por Somogyi \& Nelson (NELSON, 1944; SOMOGYI, 1945) são muito utilizadas, porém outros compostos redutores como os fenóis, taninos e flavonóides presentes na amostra representam um problema por superestimarem o conteúdo de açúcares redutores (ADRIAN et al., 2000). Os métodos cromatográficos, tais como a cromatografia líquida de alta eficiência (CLAE) e a cromatografia de gasosa (CG), proporcionam uma informação rápida e precisa sobre a composição dos açúcares, porém o elevado custo de aquisição e a manutenção dos equipamentos limita a adoção de tais métodos (ADRIAN et al., 2000).

Outra alternativa disponível, que está sendo proposta neste trabalho, é a utilização de métodos enzimáticos para a realização desta quantificação. Estes métodos são específicos, econômicos e não necessitam de equipamentos sofisticados, apenas um espectrofotômetro. A quantificação da glicose pelo método enzimático é amplamente utilizada em amostras de sangue, urina e outros líquidos biológicos (RAABO \& TERKILDSEN, 1960). Estão disponíveis no mercado "kits" laboratoriais que contêm as enzimas glicose oxidase (GOD - EC 1.1.3.4) e peroxidase (POD - EC 1.11.1.7) e 4-aminoantipirina (4-AAP). Assim, a glicose, na presença de oxigênio e da enzima GOD, é oxidada a ácido glucônico, liberando peróxido de hidrogênio, que reage com 4-AAP sob ação catalisadora da POD. O produto desta oxidação é de coloração avermelhada, sendo sua intensidade diretamente proporcional à concentração de glicose na solução, que deve ser lida em espectrofotômetro. Entretanto, o emprego deste método em amostras de batata precisa ser otimizado para evitar a interferência de outros compostos, como os fenólicos, que servem de substrato para as enzimas polifenoloxidases. Essas enzimas são responsáveis pelo escurecimento enzimático de diversos alimentos, incluindo a batata, que ocorre devido à formação de melaninas, que possuem coloração marrom (SEVERINI et al., 2003). Assim, considerando que a quantificação enzimática da glicose baseia-se na leitura da coloração em espectrofotômetro, pode-se inferir que a formação de pigmentos de coloração escura, pelas polifenoloxidases, poderia mascarar ou interferir na quantificação da glicose.

Para que uma metodologia possa ser usada rotineiramente, é importante fazer a validação da mesma, ou seja, assegurar que os requisitos para a aplicação ou o uso específico pretendidos foram atendidos (NBR ISO 9000). Assim, o objetivo deste trabalho foi adaptar e validar uma metodologia simples, econômica e eficiente para quantificar o teor de glicose em tubérculos de batata, utilizando um kit laboratorial baseado na reação com a enzima glicose oxidaseperoxidase, desenvolvido para uso em amostras de sangue e urina.

\section{MATERIAL E MÉTODOS}

Os materiais utilizados para o preparo das curvas e das amostras foram glicose anidra p.a., etanol p.a. e o kit Glucox 500 para determinação de glicose, fornecido pela empresa Doles Ltda. Foi utilizado espectrofotômetro da marca FEMTO, modelo 600S.

As amostras de batata da cultivar Macaca e Asterix foram obtidas por meio do Programa de Genética e Melhoramento da Batata do Departamento de Fitotecnia da Universidade Federal de Santa Maria. As análises foram feitas no Núcleo Integrado de Desenvolvimento em Análises Laboratoriais (NIDAL). Os tubérculos foram produzidos durante o outono e após a colheita foram deixados por 20 dias à temperatura de $25^{\circ} \mathrm{C}$ para a cura. Após, foram retirados 15 tubérculos de cada cultivar e armazenados durante 30 dias à temperatura de $8^{\circ} \mathrm{C}$.

Após o período de armazenamento, as batatas foram lavadas, descascadas e picadas. Foram pesados $4 \mathrm{~g}$ de amostra e triturados em Ultra-Turrax (modelo T18, IKA ${ }^{\circledR}$ Works Inc., Wilmington, Del., USA) com etanol absoluto pa, na proporção de 1:2 (peso/ volume). A seguir, as amostras foram centrifugadas a $2200 \times$ g durante 15 minutos e o sobrenadante foi coletado para dosagem da glicose. Utilizou-se uma alíquota de $100 \mu \mathrm{L}$ do sobrenadante, que foi misturado com $1 \mathrm{~mL}$ do reagente de cor do kit Glucox 500, o qual contém tampão fosfato $\mathrm{pH} 7,4, \mathrm{GOD}, \mathrm{POD}, 4-\mathrm{AAP}$ e phidroxibenzoato. Nessa determinação a glicose da amostra é oxidada a ácido glucônico pela enzima GOD, liberando peróxido de hidrogênio, que reage com 4AAP em reação catalisada pela POD, formando 4antipirilquinonimina. Este composto possui coloração 
avermelhada, sendo sua intensidade é diretamente proporcional à concentração de glicose na amostra. Assim, após 10 minutos de incubação a $37^{\circ} \mathrm{C}$, as amostras foram lidas em espectrofotômetro (modelo 600S, FEMTO) em $510 \mathrm{~nm}$ com cubetas plásticas de um $\mathrm{mL}$ de volume e um $\mathrm{cm}$ de comprimento óptico.

Os procedimentos seguintes foram utilizados para validar a metodologia para determinação da glicose em amostras de batata de acordo com as orientações do INMETRO no documento DOQCGCRE-008(2003).

A seletividade do método, ou seja, sua capacidade de detectar outras substâncias além do analito foi avaliada por meio da comparação das inclinações de curvas de calibração: uma curva contendo a matriz da amostra (inclui um nível básico do analito) e outra curva sem adição da amostra. As curvas foram construídas adicionando volumes crescentes de solução padrão de glicose anidra $0,1 \%$ $(0$ a $40 \mu \mathrm{L})$ a um $\mathrm{mL}$ do reagente de cor do kit Glucox 500 e procedendo a leitura conforme descrito acima. Foram feitas quatro repetições em dias diferentes, usando diferentes amostras de batata "Asterix". As curvas de calibração foram compostas por oito pontos $(0,0 ; 1,25$; 2,$5 ; 5,0 ; 10 ; 20 ; 30$ e $40 \mu \mathrm{g}$ de glicose), sendo que a curva com adição de amostra continha ainda $100 \mu \mathrm{L}$ de amostra de batata (sobrenadante obtido como descrito anteriormente). O volume final de reação de todos os pontos das duas curvas foi ajustado para $1,2 \mathrm{~mL}$ com água destilada. Os coeficientes angulares das regressões das duas curvas foram comparados via teste t de Student.

A linearidade de um método analítico corresponde a sua característica de responder proporcionalmente à adição da substância em estudo em uma dada faixa de concentração. Este parâmetro foi avaliado utilizando-se os coeficientes de correlação linear das curvas de calibração adotadas para o estudo da seletividade. Foi também aplicado um teste de hipótese para avaliar os desvios de linearidade da curva padrão (INMETRO, 2003).

O limite de quantificação representa a menor concentração do analito, que pode ser determinada com um nível aceitável de precisão e veracidade (INMETRO, 2003). O limite de quantificação foi determinado utilizando-se dez amostras de branco e o desvio padrão dessas absorbâncias e também uma curva de calibração com oito pontos $(0,0 ; 1,25 ; 2,5 ; 5,0 ; 10 ; 20 ; 30$ e $40 \mu \mathrm{g}$ de glicose) para a obtenção do coeficiente angular da regressão. A partir destes dados foi aplicada a seguinte fórmula(BRASIL, 2003):

$$
L Q=\frac{D P a \times 10}{I C}
$$

onde: $\mathrm{DPa}=$ Desvio padrão da regressão; $\mathrm{IC}=$ Coeficiente de inclinação da regressão.

As amostras de branco foram obtidas misturando-se um $\mathrm{mL}$ do reagente de cor do kit Glucox 500 com $200 \mu \mathrm{L}$ de água. A curva de calibração foi obtida conforme descrito anteriormente para a seletividade (curva de calibração sem adição de amostra).

A precisão do método proposto, que visa avaliar a dispersão de resultados entre ensaios independentes repetidos de uma mesma amostra, foi avaliada por meio dos estudos da recuperação, repetitividade e reprodutibilidade.

Para a recuperação foram fortificadas amostras com três diferentes níveis de adição de padrão, sendo uma próxima ao limite de quantificação, outra com concentração aproximada a da média da faixa de uso do método e outra próxima à concentração máxima permitida pela faixa de uso do método $(0,025 ; 0,1$; e $0,3 \mathrm{mg}$ respectivamente) em triplicata. Estas concentrações de glicose foram atingidas adicionandose volumes adequados de solução padrão de glicose $0,1 \%$ à amostra, imediatamente após a trituração da mesma no Ultra-Turrax. A recuperação foi determinada por meio da seguinte fórmula:

$$
\% \text { Recuperaçã }=\left(\frac{C_{1}-C_{2}}{C_{3}}\right) \times 100,
$$

onde: $\mathrm{C} 1$ = Concentração da amostra fortificada, $\mathrm{C} 2=$ Concentração da amostra não-fortificada e C3 = Concentração do padrão adicionado.

Para o estudo da repetitividade do método, utilizou-se um tubérculo da cultivar Macaca e a partir deste sete amostras foram preparadas de forma independente e avaliadas no mesmo dia sob as mesmas condições. A reprodutibilidade do método foi avaliada em termos da precisão intermediária. Neste estudo foi utilizada uma amostra da cultivar Macaca, que foi preparada (até a etapa de obtenção do sobrenadante) e congelada. $O$ conteúdo de glicose dessa amostra foi mensurado em sete dias diferentes, sob diferentes condições laboratoriais, variando analista e espectrofotômetro. Para determinar se os resultados da repetitividade e da precisão intermediária eram aceitáveis, utilizou-se o valor de HORRAT, que é igual ao desvio padrão relativo (RSD) obtido nas determinações de repetitividade ou na precisão intermediária, dividido pelo RSD calculado, segundo a equação de Horwitz, conforme sugere WOOD (1999). $R S D=2^{1-0,5 \log c}$ (equação de Horwitz), em que $\mathrm{C}$ é a concentração do analito na amostra.

Os resultados são considerados satisfatórios quando o valor de HORRAT é menor ou igual a dois (WOOD, 1999). 
Foi utilizado teste t de Student com um nível de erro de $5 \%$ para verificar desvios da linearidade da curva de calibração de glicose, bem como para comparar os coeficientes angulares das curvas de calibração contendo ou não a matriz da amostra.

\section{RESULTADOS E DISCUSSÃO}

O método desenvolvido demonstrou-se capaz de quantificar glicose em amostras de batata. Esse método é específico para o analito glicose, pois se trata de um método enzimático, o qual apresenta alta seletividade. A seletividade do método pode ser comprovada pelo fato de os coeficientes angulares das curvas de adição de padrão contendo ou não a matriz da $\operatorname{amostra}(0,0192$ e 0,0194, respectivamente; Figura 1A) não diferirem estatisticamente entre si, com $5 \%$ de probabilidade de erro. Estes resultados mostram que o método avaliado não apresenta componentes que possam interferir na quantificação da glicose em amostras de batata. A interferência devido à formação de produtos corados provenientes de outras reações paralelas a de quantificação da glicose, como a formação de melaninas a partir de compostos fenólicos, pela ação de polifenoloxidases, não ocorreu, possivelmente pela inibição dessas enzimas, devido à adição de etanol na etapa de trituração das amostras.

O método mostrou-se linear entre 1,25 e $40 \mu \mathrm{g}$ de glicose (Figura 1A). Os coeficientes de correlação das curvas de adição de padrão contendo ou não a matriz da amostra (0,9981 e 0,9944, respectivamente) são considerados adequados, pois estão acima de 0,90 (INMETRO, 2003). A estimativa desse coeficiente assegura a qualidade da curva obtida, pois valores próximos a um indicam uma menor dispersão do conjunto de pontos experimentais e menor incerteza dos coeficientes de regressão estimados (RIBANI et al., 2004). O teste $t$ aplicado para verificação de desvios

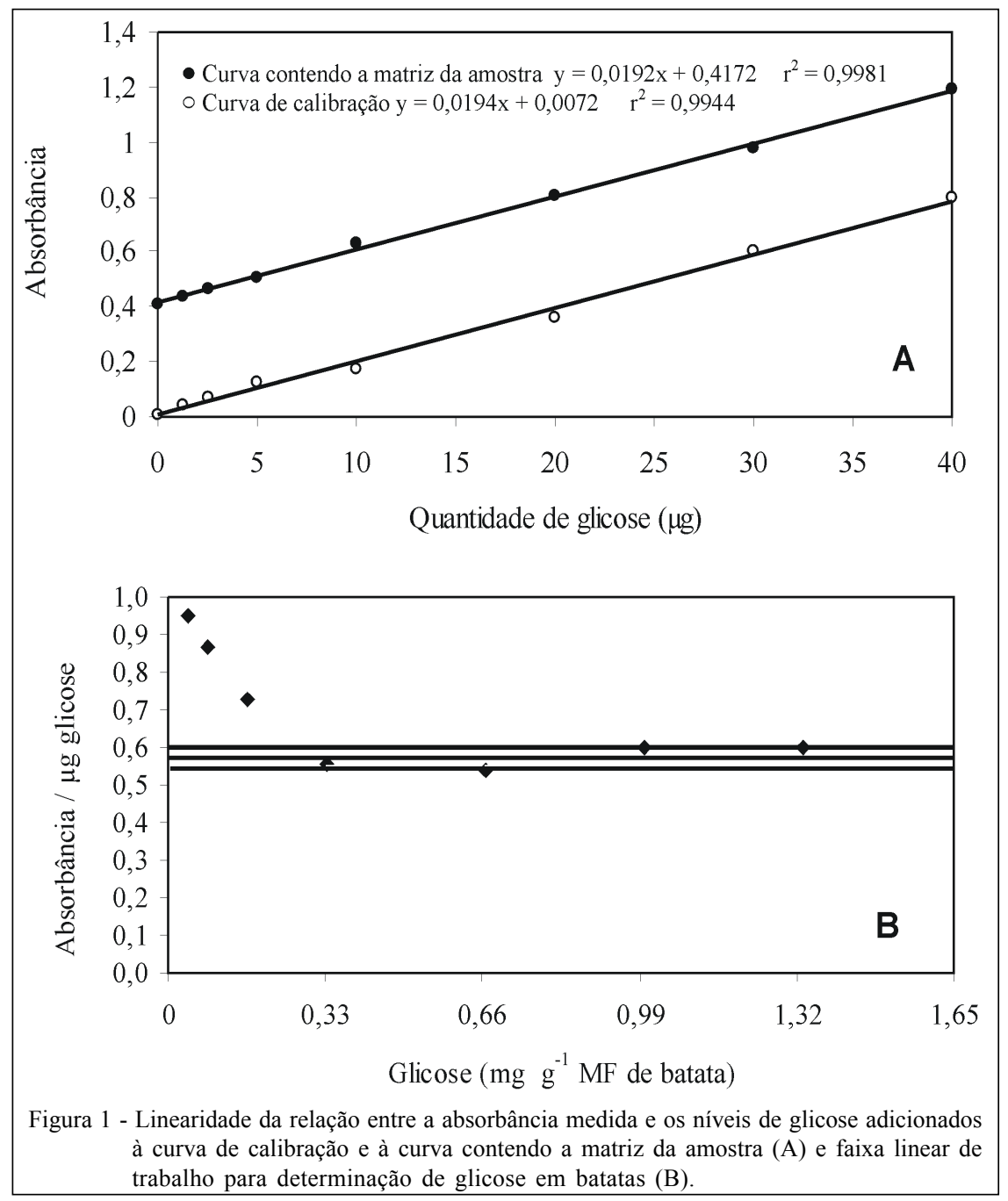

Ciência Rural, v.38, n.5, ago, 2008. 
da linearidade na curva padrão de glicose não revelou desvios estatisticamente significativos com um nível de erro de $5 \%$.

O limite de quantificação do método foi de $0,319 \mathrm{mg} \mathrm{g}^{-1}$ de massa fresca de batata. A faixa linear de trabalho (Figura 1B) apresenta o intervalo em que a resposta do sinal tem uma relação linear com o analito (glicose). Para a demonstração da faixa linear de trabalho, foi elaborado um gráfico em que são relacionados os valores relativos obtidos pela divisão da absorbância pelo nível de glicose (eixo y) versus a concentração de glicose nas amostras de batata (eixo x) (ALCAZAR et al., 2005). A partir dos pontos da faixa linear, traçou-se uma linha com inclinação zero e foram construídas ainda duas linhas paralelas representando a variação de $5 \%$ (95 e 105\%). Os pontos que pertencem à faixa linear de trabalho se encontram dentro deste intervalo de linhas paralelas, que neste caso correspondeu desde o limite de quantificação de glicose $\left(0,319 \mathrm{mg} \mathrm{g}^{-1}\right.$ de massa fresca de batata) até $1,32 \mathrm{mg} \mathrm{g}^{-1}$ de massa fresca de batata (limite superior). Amostras que contenham maior quantidade de glicose podem ser diluídas antes da análise para que fiquem dentro da faixa linear de trabalho.

No estudo da precisão do método, a recuperação média da extração foi de 99,0\% (Tabela 1), sendo esta considerada aceitável para este tipo de análise. Os valores de RSD para as avaliações de repetitividade e reprodutibilidade foram 3,63 e 3,62, respectivamente. Para a concentração de analito utilizada, o RSD calculado, segundo a equação de Horwitz, é de 5,6. Assim, considerando os RSD das análises de repetitividade e reprodutibilidade em termos de precisão intermediária, as mesmas apresentaram valores de $\operatorname{HORRAT}(0,64)$ inferiores a dois, sendo, portanto, consideradas satisfatórias.

Por meio do método proposto, os teores de glicose em batatas "Macaca" variaram entre 1,4 e 2,9mg $\mathrm{g}^{-1}$ de massa fresca (Tabela 2). Vários autores têm demonstrado que o teor de glicose em batatas pode variar entre 0,19 e $13,0 \mathrm{mg} \mathrm{g}^{-1}$ de massa fresca, quando quantificados por CLAE (EDWARDS et al., 2002), e entre um e cinco $\mathrm{mg} \mathrm{g}^{-1} \mathrm{em}$ amostra fresca, utilizando fitas indicadoras de glicose (MALLMANN, 2001).

Tabela 1 - Estudo da recuperação do método para determinação da glicose em amostras de batata

\begin{tabular}{cc}
\hline Nível de fortificação (mg) & Recuperação (\%) \\
\hline 0,025 & 100,7 \\
0,1 & 98,8 \\
0,3 & 97,3 \\
Média & 99,0 \\
\hline
\end{tabular}

Esses valores tendem a apresentar certa variabilidade devido às diferenças do material genético, manejo da lavoura e condições de armazenamento (COELHO et al., 1999; PEREIRA \& CAMPOS, 1999; MALLMANN, 2001).

O teor de glicose foi também quantificado em amostras de batata desidratadas e armazenadas por oito meses (dados não mostrados). Nesta quantificação, as amostras apresentaram conteúdo de glicose abaixo do limite de detecção do método, o que se deve provavelmente à ocorrência de reação de Maillard durante o armazenamento das amostras. Esta reação, apesar de estar positivamente relacionada com a temperatura e ser mais pronunciada acima de $50^{\circ} \mathrm{C}$, pode ser iniciada a qualquer temperatura de armazenamento (SGARBIERI, 1996; GAUCHER et al., 2008) em amostras que contenham açúcares redutores e aminoácidos, como é o caso da batata. A reação de Maillard leva à diminuição dos teores de açúcares redutores, o que a torna um fator limitante na determinação de glicose em batatas desidratadas. Além disso, o produto da reação de Maillard (melanoidinas) apresenta coloração marrom (COELHO et al., 1999; DAVIDS et al., 2004), podendo interferir na reação de cor para determinação de glicose. Assim, o modo mais adequado de armazenagem de amostras de batata para análise posterior seria a estocagem a $-20^{\circ} \mathrm{C}$ do sobrenadante obtido após homogeneização da amostra com etanol e centrifugação.

De acordo com os procedimentos de validação, não foram observadas substâncias que possam inibir a reação entre a enzima glicose oxidaseperoxidase e a glicose presente nas amostras de batata. A metodologia proposta, por ser enzimática e específica, afasta a possibilidade de que outros açúcares como a

Tabela 2 - Conteúdo de glicose em amostras de batata da cultivar Macaca.

\begin{tabular}{cc}
\hline Amostra & Glicose $^{1}$ \\
\hline 1 & 1,4 \\
2 & 1,5 \\
3 & 1,7 \\
4 & 2,3 \\
5 & 2,3 \\
6 & 2,2 \\
7 & 2,9 \\
8 & 2,8 \\
9 & 2,9 \\
10 & 2,7 \\
11 & 2,8 \\
12 & 2,7 \\
\hline
\end{tabular}

${ }^{1}$ Glicose em mg g ${ }^{-1}$ massa fresca de batata.

Ciência Rural, v.38, n.5, ago, 2008. 
frutose e também outras substâncias participem da reação e superestimem os valores encontrados.

\section{CONCLUSÕES}

O método enzimático desenvolvido para a quantificação espectrofotométrica do teor de glicose em amostras frescas de batata é prático, econômico e altamente específico para o analito. Os resultados indicam que a metodologia apresenta seletividade, linearidade e precisão para ser utilizada pelos programas de melhoramento que visam selecionar clones com baixos teores de açúcares redutores.

\section{AGRADECIMENTOS}

Os autores agradecem a empresa Doles Reagentes e Equipamentos para Laboratórios Ltda. (Goiânia, GO), pela doação dos kits Glucox 500 para realização deste estudo. Ao Conselho Nacional de Desenvolvimento Científico e Tecnológico (CNPq), pela concessão de bolsa de Produtividade em Pesquisa aos autores Tatiana Emanuelli e Dilson Antônio Bisognin. Ao PIBIC/CNPq, pela concessão de bolsa à Engil Isadora Pujol Pereira, durante o desenvolvimento deste trabalho. À Coordenação de Aperfeiçoamento de Pessoal de Nível Superior (Capes/Fulbright), pela concessão de bolsa a Sérgio Tonetto de Freitas.

\section{REFERÊNCIAS}

ADRIAN, J. et al. Análisis nutricional de los alimentos. Zaragoza: Acribia, 292p., 2000.

ALCAZAR, A. et al. Enzymatic-spectrophotometric determination of sucrose in coffee beans. Talanta, v.67, p.760$766,2005$.

BRASIL. Ministério da Saúde. Secretaria de Vigilância Sanitária. Resolução RE n'899, de 29 de maio de 2003. Determina a publicação do Guia para validação de métodos analíticos e bioanalíticos. Acesso em: 30 de julho de 2007. On-line. Disponível em: <http://www.anvisa.gov.br/legis/resol/2003/re/ 899_03re.htm $>$.

BROWN, J. et al. The processing potential of tubers of the cultivated potato, Solanum tuberosum L., after storage at low temperatures. 2. Sugar concentration. Potato Research, Wageningen, v.33, p.219-227, 1990.

COELHO, A.H.R. et al. Qualidade de batata (Solanum tuberosum L.) para fritura, em função dos níveis de açúcares redutores e amido, durante o armazenamento refrigerado e à temperatura ambiente com atmosfera modificada. Ciência e Agrotecnologia, v.23, p.899-910, 1999.

DAVIDS, S.J. et al. Use of storage temperatures to improve the amino acid profile of potatoes for novel flavoring applications. Lebensm-Wiss u-Technology, v.42, n.2, p.1-8, 2004.
EDWARDS, C.G. et al. Changes in color and sugar content of yellow-fleshed potatoes stored at three different temperatures. Short Communication American Journal of Potato Research, v.79, p.49-53. 2002.

SEVERINI, C. et al. Prevention of enzymatic browning in sliced potatoes by blanching in boiling saline solutions. Lebensm-Wiss u-Technology, v.36, n.7, p.657-665, 2003.

GAUCHER, I. et al. Effects of storage temperature on physicochemical characteristics of semi-skimmed UHT milk. Food Hydrocolloids, v.22, n.1, p.130-143, 2008.

Instituto Nacional de Metrologia, Normalização e Qualidade Industrial (INMETRO). Orientações sobre validação de Métodos de Ensaios Químicos, Rio de Janeiro, DOQ-CGCRE008, 35p., 2003.

MALLMANN, N. Efeito da adubação na produtividade, qualidade e sanidade de batata cultivada no centro-oeste paranaense. 2001. 151f. Dissertação (Mestrado em Agronomia) - Programa de Pós-graduação em agronomia, Universidade Federal do Paraná.

MARQUEZ G.; AÑON, M.C. Influence of reducing sugars and amino acids in the color development of fried potatoes. Journal of Food Science, v.51, p.157-160, 1986.

NBR ISO/IEC 9000:2000. Sistemas de gestão da qualidade Fundamentos e vocabulário. Rio de Janeiro, ABNT. 3p., 2000.

NELSON, N. A fotometric adaptation of Somogyi method for the determination of glucose. Journal of Biological Chemistry, v.153, p.375-380, 1944.

PEREIRA, A. da S.; CAMPOS, A. Sugar content in potato (Solanum tuberosum L.) genotypes. Ciência Rural, v.29, p.13-16, 1999.

PEREIRA, A. da S. et al. Potential for improvement by selection for reducing sugar content after cold storage for three potato populations. Theoretical and Applied Genetics, v.88, p.678-684, 1994.

RAABO, E.; TERKILDSEN, T.C. On the enzymatic determination of blood glucose. Scandinavian Journal of Clinical and Laboratory Investigation, v.12, p.402-407, 1960 .

RIBANI, M. et al. Validation for chromatographic and electrophoretic methods. Química Nova, v.27, p.771-780, 2004.

SGARBIERI, V.C. Proteínas em alimentos protéicos: Propriedades, degradações e modificações. São Paulo: Varela, 1996. 517p.

SOMOGYI, M. A new reagent for determination of sugars. Journal of Biological Chemistry, v.160, p.61-68, 1945.

WOOD, R. How to validate analytical methods. Trends in Analytical Chemistry, v.18, p.624-632, 1999. 